CERN-PPE/95-64

21 April 1995

\title{
Performance of an Electromagnetic Liquid Krypton Calorimeter based on a Ribbon Electrode Tower Structure
}

G. D. Barr, C. Bruschini, G. Bocquet, P. Buchholz 1), D. Cundy, N. Doble, W. Funk, L. Gatignon, A. Gonidec, B. Hallgren, G. Kesseler, A. Lacourt, G. Laverrière, G. Linser, M.Martini, A. Norton, D. Schinzel, W. Seidl, M.S. Sozzi, H. Taureg, O. Vossnack, H. Wahl, P. Wertelaers, J. Weterings, and M. Ziolkowski

CERN, 1211 Geneva 23, Switzerland

A. Kalinin, V. Kekelidze and Yu. Kozhevnikov

Joint Institute for Nuclear Research, Dubna, Russia

L. Bertolotto

Department of Physics and Astronomy, University of Edinburgh, U.K.

V. Carassiti, J. Duclos, A. Gianoli, P.L. Frabetti ${ }^{2)}$, and M. Savriè

Dipartimento di Fisica dell'Università e Sezione dell'INFN di Ferrara, 44100 Ferrara, Italy

O. Zeitnitz

Institut für Physik, Universität Mainz, 55099 Mainz, Germany ${ }^{3)}$

M. Calvetti, P.Lubrano and M. Pepe

Dipartimento di Fisica dell'Università e Sezione dell'INFN di Perugia, 06100 Perugia, Italy

P. Calafiura ${ }^{4)}$, C. Cerri, F.Costantini, R. Fantechi, B. Gorini, I. Mannelli, and V. Marzulli Dipartimento di Fisica dell'Università e Scuola Normale Superiore e Sezione dell'INFN di Pisa, 56010 Pisa, Italy

D. Bédérède, P. Debu, A. Givernaud, L. Gosset, J. Heitzmann, E. Mazzucato, B. Peyaud and R. Turlay

CE-Saclay-DAPNIA, 91191 Gif-sur-Yvette, France

C. Bïno, A. Ceccucci, P. Maas and S. Palestini

Dipartimento di Fisica Sperimentale dell'Università e Sezione dell'INFN di Torino, 10125

Torino, Italy

Cagliari-Cambridge-CERN-Dubna-Edinburgh-Ferrara-Mainz-Orsay

Perugia-Pisa-Saclay-Siegen-Torino-Vienna Collaboration

(To be submitted to NIM)

\footnotetext{
1) Present address : Institut für Physik, Universität Mainz, 55099 Mainz, Germany

2) Permanent address: Dipartimento di Fisica dell'Universitá di Bologna, 40126 Bologna, Italy

3) Funded by German Federal Minister for Research and Technology (BMBF) under contract 054 MZ18.
} 


\section{Abstract}

The NA48 collaboration is preparing a new experiment at CERN aiming to study CP violation in the $K^{0}-\overline{K^{0}}$ system with an accuracy of $2 \times 10^{-4}$ in the parameter $\mathcal{R} e\left(\epsilon^{\prime} / \epsilon\right)$. Decays into two $\pi^{0}$ 's will be recorded by a quasi-homogeneous liquid krypton calorimeter. A liquid krypton calorimeter has been chosen to combine good energy, position and time resolution with precise charge calibration and long-term stability. The prototype calorimeter incorporating the final design of the electrode read-out structure is presented in this

paper. An energy resolution of $\simeq \frac{3.5 \%}{\sqrt{E}}$ with a constant term smaller than $0.5 \%$ has been obtained. The time resolution was found to be better than 300 ps above $15 \mathrm{GeV}$. 
The NA48 collaboration is preparing a new experiment to measure $\mathcal{R} e\left(\epsilon^{\prime} / \epsilon\right)$ with an accuracy of $2 \times 10^{-4}$ [1]. In order to reduce the statistical errors, a tenfold increase in beam intensity over the previous experiment at CERN [2] is planned. To keep the magnitude of the statistical and systematic errors similar, as in the previous experiment, a reduction of the systematic uncertainties by a factor of three is also needed. The principle of the experiment is to collect the four relevant decay modes $\left(K_{L}^{0} \rightarrow \pi^{+} \pi^{-}, K_{L}^{0} \rightarrow \pi^{0} \pi^{0}\right.$, $\left.K_{S}^{0} \rightarrow \pi^{+} \pi^{-}, K_{S}^{0} \rightarrow \pi^{0} \pi^{0}\right)$ concurrently, in the same detector using almost collinear $K_{L}$ and $K_{S}$ beams, distinguished by tagging the proton which produces the $K_{S}$ particle [3]. The charged decays will be reconstructed by a magnetic spectrometer. To reconstruct the neutral decays, a good electromagnetic calorimeter is needed. A quasi homogeneous liquid krypton (LKr) ionization chamber has been chosen to combine good energy, position and time resolution with precise charge calibration and long-term stability. The tower structure geometry is essential to guarantee good time resolution, to minimize the effect of overlapping showers from pile up events and to reduce the ambiguity in pairing photons coming from the same $\pi^{0}$. In addition a tower read-out structure permits low capacitance detector channels, allowing fast ionization charge read-out, essential to cope with the $\simeq 1$ $\mathrm{MHz}$ singles rate.

The prototype calorimeter consists of a matrix of 184 cells formed by thin electrodes stretched longitudinally. One elementary cell is $2 \times 2 \mathrm{~cm}^{2}$ in size; each cell is formed by two drift gaps sharing the collection electrode and read out by the same preamplifier. Each drift gap is $10 \mathrm{~mm}$ wide, corresponding to a drift time of about $2.8 \mu \mathrm{s}$ for a bias high voltage (HV) of $5000 \mathrm{~V}$. The active krypton length is $125 \mathrm{~cm}$ corresponding to about $27 X_{0}$. Three different electrode structures were tested. The first two, based on thin Kapton foils with glued copper electrodes, are described in a previous paper [4], where details about the cryogenics and the gas purification system can also be found. These earlier prototypes proved that the LKr approach is adequate for the experiment in terms of energy, position and time resolution, but also underlined the inherent difficulties in realizing a sufficiently uniform electrode tower structure scalable to the full-size detector. In this paper we present the results achieved with a prototype calorimeter incorporating the electrode structure chosen for the full size NA48 detector.

\section{$2 \quad$ Read-out technique}

A charge $q$ drifting with velocity $\overrightarrow{v_{d}}$ induces on a given electrode a current:

$$
I=q \overrightarrow{v_{d}} \cdot \vec{P}
$$

where $\vec{P}=\vec{E} / V_{0} \cdot \vec{E}$ is the electric field that would be present at the location of the drifting charge when the electrode is maintained at a potential $V_{0}$ while all other electrodes are grounded. In general $\vec{P}$ and $\overrightarrow{v_{d}}$ depend on the geometry of the electrodes and the location of the drifting charge. Since in LKr only the electrons drift with appreciable velocity, the contribution to the induced signal due to the ions is negligible. As a consequence, for a given amount of deposited ionization, the integration of the induced current over the drift time yields a charge that fluctuates as a function of how the ionization is deposited across the drift gap. In order to minimize such fluctuations the initial current is measured [5]. A short shaping time is needed to reduce the variations but a too short shaping time would deteriorate the signal to noise ratio. This is particularly important in a device without gain like an ionization calorimeter where many cells are added together to extract the 
information. The charge preamplifier, based on an original design from V. Radeka et al. [6] is used in the LKr, directly coupled to the electrode structure by means of a blocking $H V$ capacitor $C_{b}{ }^{1}$. The signals then leave the cryostat and are received by a $(C R)^{2}-(R C)^{4}$ shaping amplifier [7] which provides an output pulse proportional to the derivative of the current averaged over $\simeq 100 \mathrm{~ns}$. The pulses are digitized by 12 bit peaksensing ADCs ${ }^{2)}$ and, in parallel, by 8 bit FADCs ${ }^{3)}$. In addition, during a test beam run in 1994, 8 cells were equipped with prototypes of the read-out electronics planned for the NA48 experiment. The results obtained by means of the prototype electronics, are reported in section 5.6. The krypton radioactivity produces a small DC current ${ }^{4)}$ but it does not contribute significantly to the electronics noise.

It is worth recalling why a tower ionization calorimeter needs a geometrically accurate electrode structure. The transverse shower profile is rather narrow and very steep near its axis (up to $50 \%$ of the shower energy can be deposited in the calorimeter cell which is hit by the showering particle). If we consider the ionization associated with the shower core $q_{c}$ to be locally deposited between parallel plane electrodes separated by a distance $W$, the induced initial current from equation 1 becomes

$$
I=q_{c} \overrightarrow{v_{d}} \cdot \frac{\vec{E}}{V_{0}}=q_{c} v_{d} \cdot \frac{E}{E \cdot W}=q_{c} \frac{v_{d}}{W}
$$

clearly depending upon the drift gap even in the case of an applied HV high enough to produce a saturated drift velocity. This dependence would vanish in the case of uniform density of the ionization over the distance of several drift gaps. This is not an appropriate approximation for our case, as shown by test data and Monte Carlo simulation. In fact in order to keep the energy independent term of the resolution within $0.5 \%$, the tolerance on the (nominal $1 \mathrm{~cm}$ ) drift gap must be smaller than $100 \mu \mathrm{m}[8]$. The effect of inaccuracy in the semi-cell drift gap is clearly visible in the data collected with the Kapton foil electrode structure [4].

\section{$3 \quad$ The electrode read-out structure}

To solve the non-uniformity problems described above, a completely new electrode structure has been designed, constructed and tested. The read-out structure is based on ribbon electrodes stretched between two end-plates. The electrodes are $1268 \mathrm{~mm}$ long, $18 \mathrm{~mm}$ wide, and $40 \mu \mathrm{m}$ thick, and are made of a copper-beryllium alloy. Each cell is formed by a central anode and by two cathodes at $10 \mathrm{~mm}$ distance on each side of the anode. The cathodes are shared between horizontally adjacent cells. Vertically, the electrodes are aligned along planes set at the same voltage. The distance between electrodes in the vertical direction is $2 \mathrm{~mm}$, so that the transverse size of each cell is $20 \times 20 \mathrm{~mm}^{2}$. Longitudinally, the correct spacing between the electrodes is enforced by five slotted plates. These spacer-plates push the ribbons alternatively from the right and the left side, positioning them at angles of about $\pm 50 \mathrm{mrad}$ from the detector axis. The detector consists of 184 cells, formed by 383 ribbons. An artist's view of the electrode read-out structure is shown in figure 1. The zig-zag applied to the geometry of the ribbons plays two roles. Firstly, it

1) $13000 \mathrm{~V}, 3 \mathrm{nF} \pm 10 \%$, produced by ASC Corporation, Ogallala, Nebraska, US

2) LeCroy Research System, Model 2281.

3) Dr. Bernd Struck, STR750 8-bit Flash-ADC system.

4) In the test beam setup with the 180 litres prototype cryostat full of krypton, a DC dark current of $250 \mathrm{nA}$ was measured, mostly explained by the krypton radioactivity. 


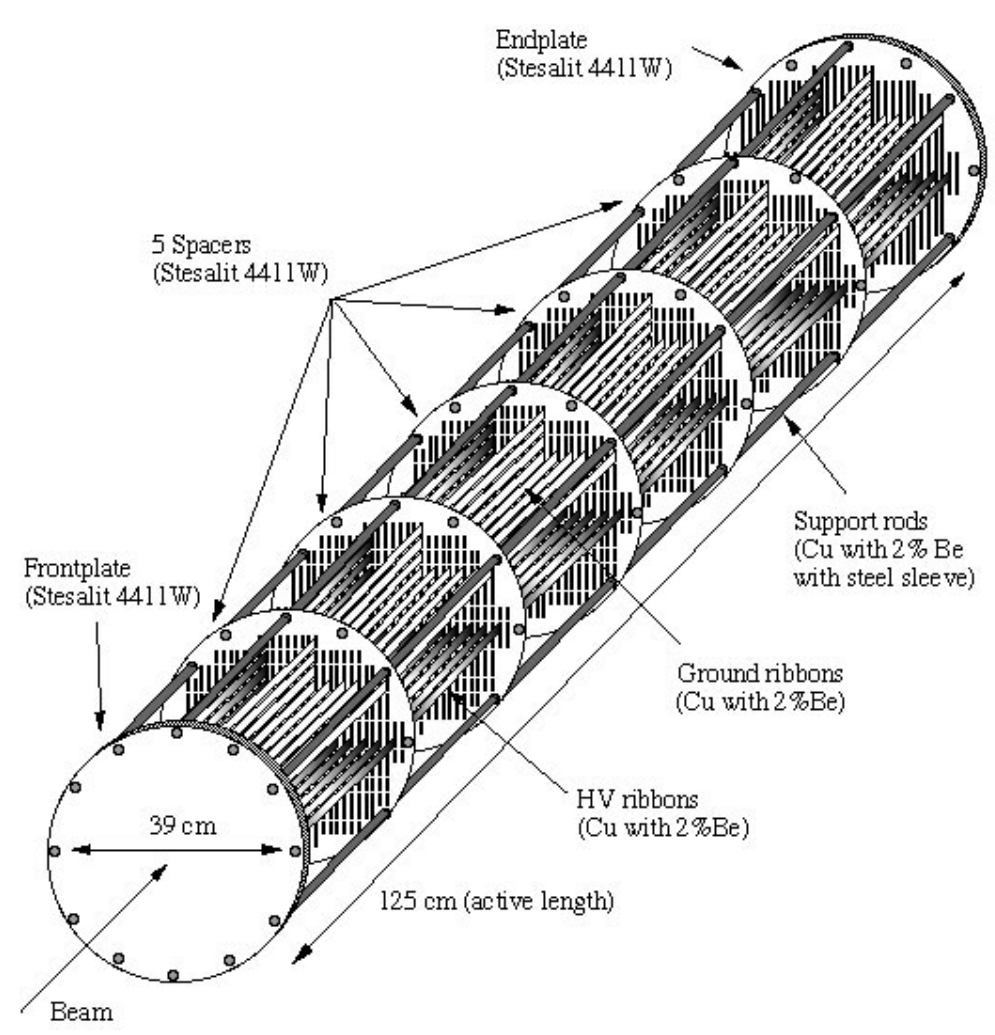

Figure 1: The prototype electrode structure.

effectively reduces the length over which the electrodes are stretched from 1270 to $208 \mathrm{~mm}$, allowing to maintain their correct spacing against electrostatic forces with a small tension. The good precision in the manufacturing of ribbons and spacer-plates allows to achieve a satisfactory uniformity of the cell geometry. Secondly, the read-out cells are arranged in a tower geometry without having the electrodes parallel to the direction of the impinging particles, which is a significant advantage, as discussed in the following. The passive material at the electrodes, and to a greater extent the finite integration time needed for the read-out of the initial current, lead to a loss of response for ionization deposited in the proximity of the electrodes: namely, if $\Delta t$ is the measuring time used to average the initial current, a charge $q$ deposited closer to the anode than $v_{d} \cdot \Delta t$, contributes only partially to the current. Electromagnetic showers in krypton are characterized by a narrow core which, if placed close to an anode, would generate a smaller signal for the full shower. The zig-zag geometry reduces the depth inside the detector along which a shower core may be close to an electrode. In particular, in our geometry an electrode moves transversally to the beam direction by $10 \mathrm{~mm}$, equal to the full length of an anode-to-cathode gap, along the depth of $208 \mathrm{~mm}$ separating two spacer-plates.

\subsection{Electrodes}

The electrodes are made with a copper-beryllium alloy. This choice follows a period of studies during which we tried to produce ribbons made with plastic films coated with metals. Commercially available metallized polyester films are not suited for our application because the thickness of the conductor is of the order of $0.1 \mu \mathrm{m}$, and the resistance of the electrodes would be large enough to affect the performance of the calorimeter. Ribbons made of cold rolled metal tapes appeared to be easier to manufacture and more 
reliable in terms of maintaining geometrical accuracy after cool-down to liquid krypton temperature. A copper alloy is chosen because it does not undergo phase transformations at low temperature. The small quantity ( $2 \%$ ) of beryllium makes the metal harder, and therefore less susceptible to geometrical damage during handling, and suited to be used as an elastic device, once it is given the shape of a spring, as discussed below.

Ribbons were delivered ${ }^{5)}$ with good geometry (flatness to $\pm 0.05 \mathrm{~mm}$, width accurate to about $\pm 0.01 \mathrm{~mm}$, straightness of edge within $\pm 0.1 \mathrm{~mm}$ ). The tensile force applied to each electrode is equal to $2-2.5 \mathrm{~N}$. This force allows to operate the calorimeter with high voltages up to $10 \mathrm{kV}$ with little effects on the electrode geometry. The elasticity of the ribbon is small, such that under $2 \mathrm{~N}$ of tension, the ribbon length increases by only $0.03 \mathrm{~mm}$. The distance between end-plates is defined by $\mathrm{Cu}$-Be rods, surrounded by cylindrical shells made of stainless steel defining the distance between spacer-plates. The difference in thermal contraction between rods and ribbons is therefore at least partially compensated. However, to provide the necessary stability and uniformity in tensioning the ribbons, compliant elements are needed. After a number of trials, the solution was found in the form of accordion-like springs, at the two ends of the ribbons close to the end-plates. These devices are also important in allowing less tight tolerances in the positioning and orientation of the ribbons at the end-plates.

Near the back-plate, the springs are formed by $\mathrm{Cu}$-Be ribbons pressed into an approximately sinusoidal shape. The springs are $27 \mathrm{~mm}$ long, with a transverse peak-to-peak amplitude of about $2 \mathrm{~mm}$. The elastic constant is equal to about $1.0 \mathrm{~N} / \mathrm{mm}$ (and about $10 \%$ stiffer at liquid nitrogen temperature). The springs are subjected to a thermal hardening treatment, and measured to be linear up to about $4 \mathrm{~N}$ of tension. The springs are soldered to the ribbons. Thanks to these springs, the tension applied to the ribbons is less sensitive to differences in the distances between end-plates at room and liquid krypton temperature.

Similar springs are also needed near the front-plate. They have the same shape of the back springs, but are roughly a factor of two shorter and stiffer. The main purpose of these is to reduce the sensitivity to the positioning of the ribbons at the front-plate. Inaccuracies are absorbed by the springs without appreciably affecting the tensioning profile applied over the width of the ribbons. A ribbon is sketched in figure 2 .

\subsection{End-plates and spacer-plates}

End-plates and spacer-plates are machined out of fiberglass-epoxy composite. ${ }^{6)}$ This material is chosen because it allows accurate machining, it has good in-plane isotropy with respect to thermal contraction, it has high values of mechanical stiffness and strength despite a relatively long radiation length, and it does not affect the lifetime of drifting electrons in liquid krypton. The end-plates are $50 \mathrm{~mm}$ thick and the spacer-plates $5 \mathrm{~mm}$.

The front-plate is machined with grooves, in the shape of a half dove tail. Each electrode (ribbon) is glued to a piece (front-domino) machined out of the same material as the plates, which fits the shape of the groove. The front-dominos are piled up in the grooves.

At the other end, the ribbon is fixed, by means of soldering and two screws, to a piece made of brass (back-domino), which fits into a rectangular groove machined in the back-plate. A thin brass rod, partially threaded and gold plated, is connected to the

5) Matthey S.A., CH 2520 La Neuveville.

6) $4411 \mathrm{~W}$, manufactured by Stesalit A.G., CH Zullwil. 


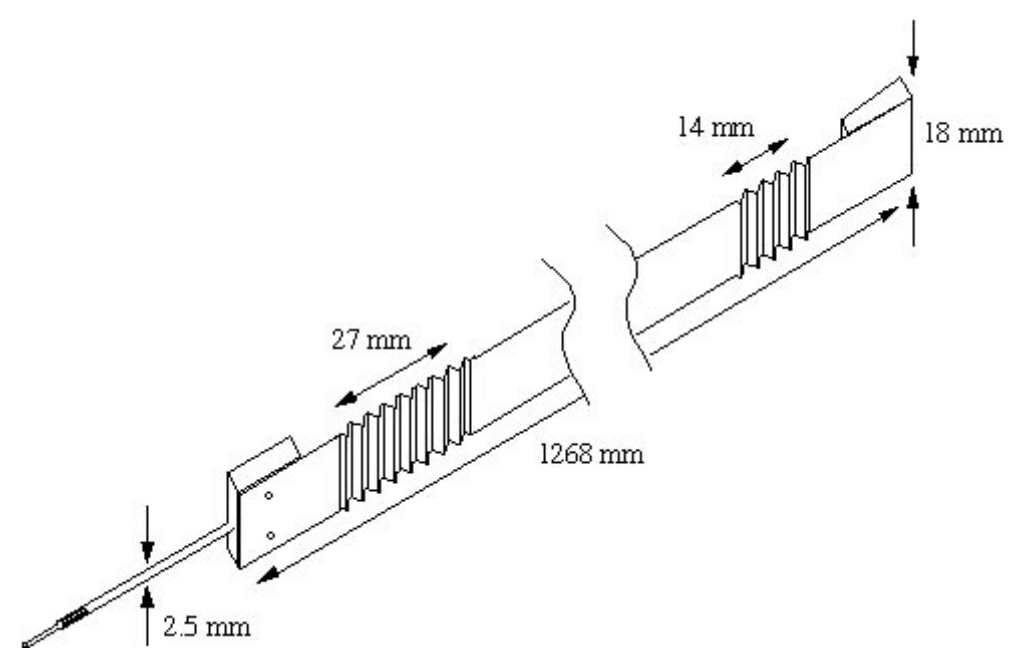

Figure 2: Ribbon design.

domino on the back side, and is inserted into a hole through the back-plate, reaching the outside. There the longitudinal position of the domino is fixed with a nut, and the tip of the rod acts as an electrical contact to the HV biasing and to the read-out electronics.

The spacer-plates are machined with slots covering almost $50 \%$ of the surface of the plates. The slots are $18.5 \mathrm{~mm}$ high, and $5 \mathrm{~mm}$ wide in order to allow the passage of back dominos during the assembly of the electrodes. The pitch between the sides of the slots against which the ribbons rest, is machined to an accuracy of $\pm 0.02 \mathrm{~mm}$. The thickness of these plates was chosen with the full-size NA48 detector with a cross section 70 times larger than the prototype in mind. Studies based on Monte Carlo simulations show that the thickness of the spacer-plates is small enough not to affect the performance of the detector. Similarly, computations and simulations of electric fields show that the front- and back-spring do not deteriorate the performance of the device.

\subsection{Connection to the front-end electronics}

The front-end electronics is coupled to the electrode structure in a completely modular way. The electrode structure is fully assembled without interference from the HV distribution system or the front-end circuitry. The electrical connection between the HV distribution system and the ribbons is realized by the thin brass rod described in section 3.2. The tip of the rod is machined to the shape of a pin suitable for mating to commercial electrical contacts ${ }^{7}$. Such contacts are housed in properly machined boards made of the same epoxy composite used for the end plates and the spacers in order to avoid differential thermal contractions. The HV distribution circuitry and the blocking capacitors are attached to these boards which connect up to 32 channels. The HV is distributed to each tower by means of a $10 \mathrm{M} \Omega$ resistor; this value has been chosen sufficiently high to damp microphonics oscillations and, at the same time, small enough to limit the voltage drop during the beam spill to a few volts. The front-end boards, holding the preamplifiers, are attached to the HV distribution system by means of the same type of contacts used to connect the HV distribution system to the electrode structure. This modular scheme permitted the replacement of the HV distribution system ${ }^{8}$, during a dedicated test beam

7) FCI BURNDY machined contacts type RC16M23K.

8) A scheme without blocking capacitors and without blocking resistors was tested [9]. 
period, without modifications to the electrode read-out structure or to the front-end electronics.

\subsection{The calibration system}

In principle, the calibration of a LKr calorimeter is simple, since the gain stages (charge preamplifier and shaping amplifier) and the read-out electronics can be precisely calibrated by charge injection. Calibration pulses of initial current values determined by an input DC voltage are produced with a DMOS-FET switch and a RC circuit on the preamplifier motherboard. All the capacitances and resistances of the calibration system were measured at room temperature and at liquid nitrogen temperature. By interpolation, they are known at LKr temperature to better than $1 \%$ accuracy. The elements that cannot be calibrated by the charge injection at the level of the preamplifier are the electrode structure imperfections and the spread of the blocking capacitance $C_{b}$. The spread of $C_{b}$ must be small since the blocking capacitor attenuates the measured charge by $\simeq 6 \%$. For the prototypes, the HV blocking capacitors were measured and only the ones found to be within $\pm 1.5 \%$ of the average are used.

\section{$4 \quad$ Test beam setup}

The prototype calorimeter was installed in the H4 beam line at the CERN-SPS, where it was exposed to an electron beam. Data were collected with energies ranging from 8 to $80 \mathrm{GeV}$. The momentum bite of the electron beam was defined by collimation to be $\sigma(E) / E=0.5 \% / \sqrt{(} E)$. In order to measure the position resolution of the calorimeter, the electron impact point is reconstructed with a precision of about $100 \mu \mathrm{m}$ by two small drift chambers. The total amount of passive material in front of the active krypton was about $0.6 X_{0}$. The trigger was given by two large scintillator counters, in front of the calorimeter. For the time measurement, the time reference was given by a smaller scintillation counter. A veto system covered the region outside the acceptance of the two drift chambers and of the thin entrance window.

\section{$5 \quad$ Results}

The results presented in this paper regarding position and energy resolution, uniformity of the response, drift time measurements and the response to high intensity, are obtained with data collected during a test beam period in November 1993. The results presented in section 5.6, concerning the time resolution, are based on data collected in September 1994.

\subsection{Position resolution}

The space resolution of the prototype calorimeter is obtained by comparing the position of the center of gravity ( $\mathrm{COG}$ ) of the shower in the calorimeter with the electron impact point as reconstructed by the drift chambers. The COG is calculated using a $3 \times 3$ shower box and corrected for the non-linear dependence on the impact point within a cell. The space resolution as a function of the beam energy is shown in figure 3 and is parameterized as:

$$
\begin{aligned}
\sigma_{x} & =\left(\frac{4.2}{\sqrt{E[G e V]}} \oplus 0.6\right) m m \\
\sigma_{y} & =\left(\frac{4.3}{\sqrt{E[G e V]}} \oplus 0.6\right) \mathrm{mm}
\end{aligned}
$$




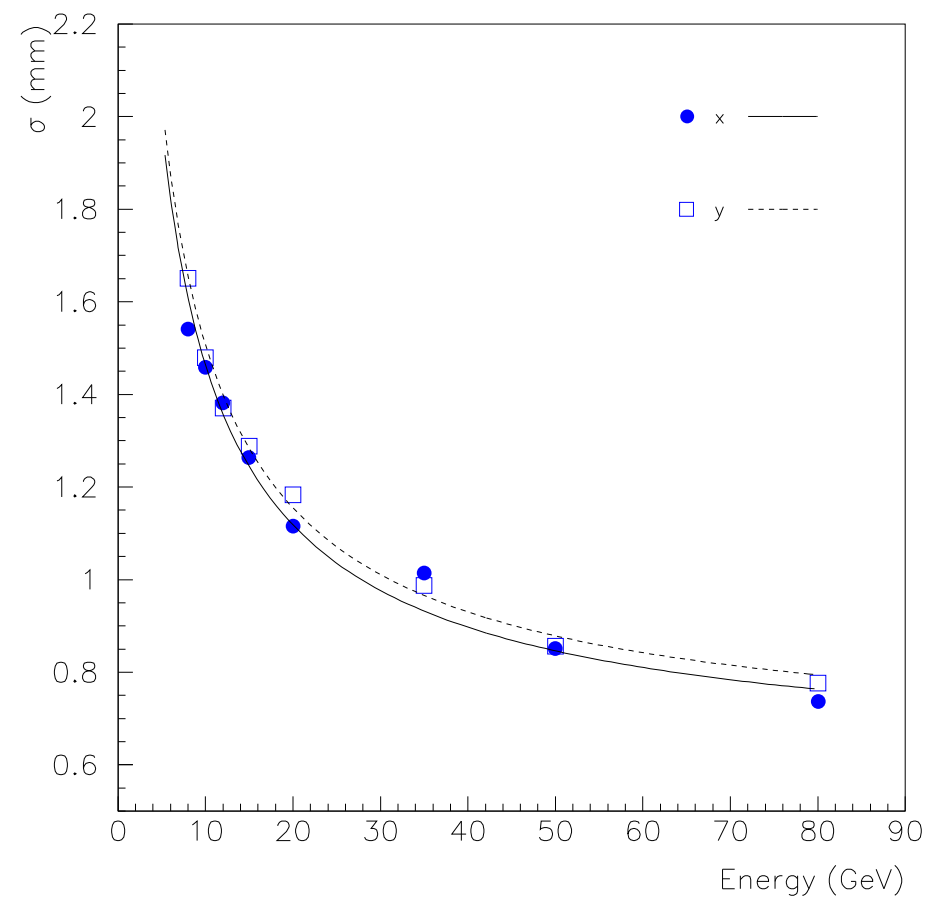

Figure 3: Space resolution as function of the electron beam energy.

\subsection{Energy Resolution}

The factor used to convert ADC counts to initial current in $\mu \mathrm{A}$ is determined using the calibration circuit described in section 3.4. Pulse samples of different amplitudes give the ADC to current conversion over the range of interest. A pulse-height spectrum for 20 $\mathrm{GeV}$ electrons, before any correction, is shown in figure 6 . The following cuts are applied to the data sample: the difference between the total energy in the calorimeter and the energy contained in an $11 \times 11$ shower box centered on the cell containing the maximum energy is required to be consistent with the expected leakage outside the shower box for electrons; the cluster width is also required to be consistent with that expected for electrons. These cuts serve to remove the small $(\leq 1 \%)$ muon and pion contamination of the electron beam.

The $11 \times 11$ shower box response is corrected as a function of the impact point within the cell (as reconstructed by the calorimeter) by an uniformity correction common to all the cells and slightly energy dependent. The magnitude of the uniformity corrections is within $\pm 1 \%$ as can be seen in figure 4 . The non-uniformity at the $y$ boundaries of the read-out cell is due to the $2 \mathrm{~mm}$ vertical gap between the electrodes. The dip structure seen as a function of $x$ is due to the finite shaping time: the position of the minimum indicates the impact point at which the shower core crosses (at the depth of the shower maximum) the collection electrode. A faster shaping would reduce the effect. The $x$ and $y$ corrections are applied independently. Cell to cell variations, for example due to geometrical imperfections, are not corrected (see section 5.4 for a quantitative study). The resolution is defined as the ratio of the width to the mean $\left(\frac{\sigma}{\mu}\right)$ of a Gaussian fit to the measured current distribution summed in a shower box of $11 \times 11$ cells around the shower maximum. Given the limited lateral size of our prototype an $11 \times 11$ shower box can be 

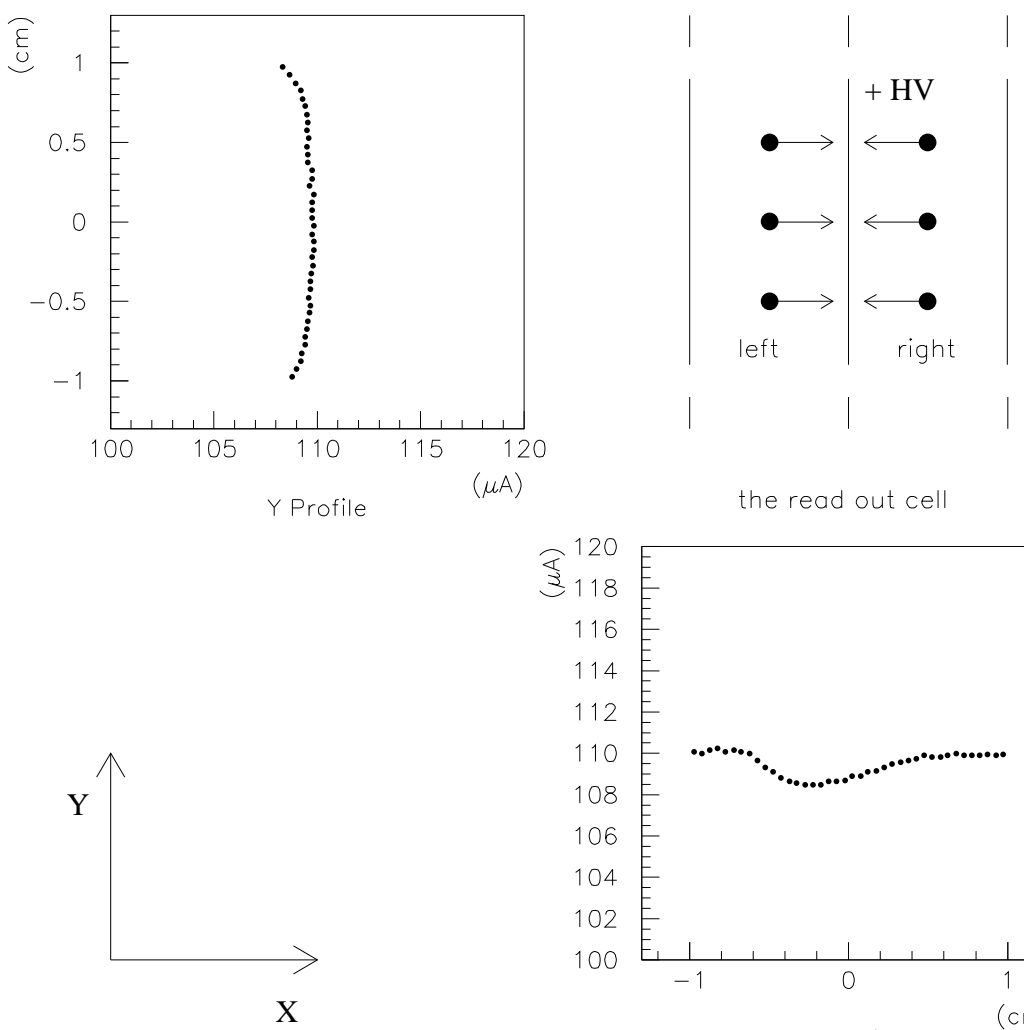

the read out cell

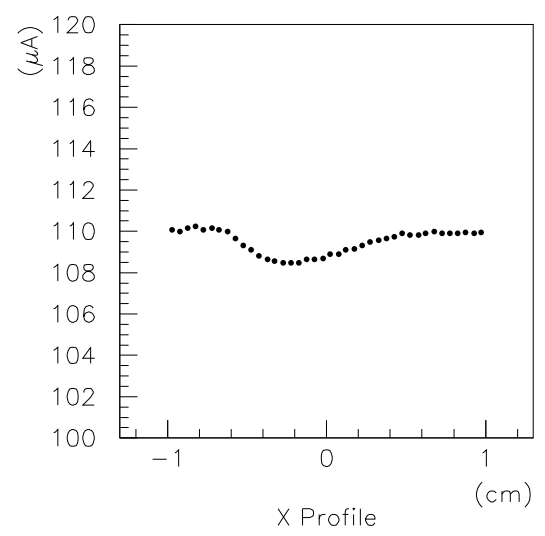

Figure 4: Non-uniformities of response as a function of the impact point. 
calculated for 2 cells only, but neglecting the contribution from two extreme corners, the $11 \times 11$ shower box can been defined for 12 cells as shown in figure 5 . For this reason the

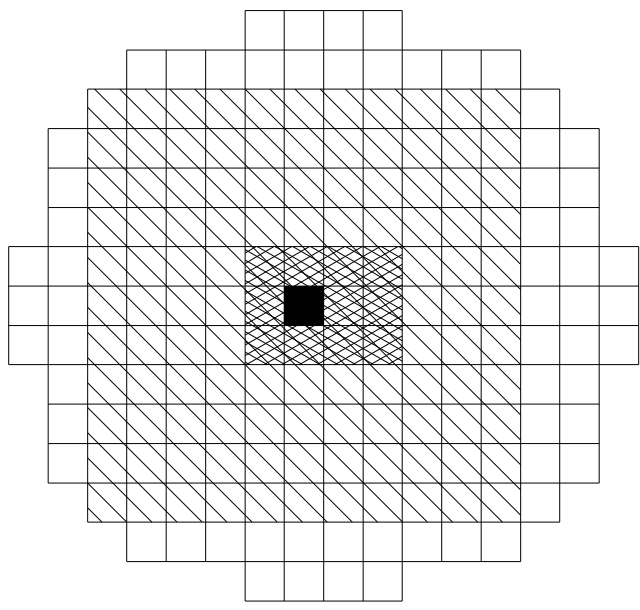

Figure 5: An $11 \times 11$ shower box (hatched) can be defined around a hit cell (black) belonging to the 12 central cells (cross hatched).

analysis requires the electrons to hit one of the twelve central cells of the calorimeter. The energy resolution as a function of the electron energy is shown in figure 7 . The statistical term of the superimposed curve is $3.5 \% / \sqrt{E}$. The noise contribution (about $40 \mathrm{MeV}$ ) is evaluated from the calibration data fluctuations. The constant term is consistently smaller than $0.5 \%$ demonstrating that the non-uniformity problems observed in the previous prototypes [4], have been solved thanks to the built-in geometrical accuracy of the ribbon electrode structure.

\subsection{Comparison with Monte Carlo expectations}

A detailed Monte Carlo simulation [10] has been performed to study specific issues of the detector design. The simulation is based on a full GEANT description of the electrode structure and of the electromagnetic shower development. A realistic electric field map is used. The simulation includes the signal induction on the electrodes and the behaviour of the read-out components. To verify our understanding of the device, we compare the test beam results with the Monte Carlo predictions. To make the comparison possible, the Monte Carlo and the beam test data sets need to be analyzed in the same way and, for the test beam data, the best possible calibration constants should be used. To this purpose, a subset of the data taken at each energy has been used to calculate correction factors to the calibration constants for the eighteen central cells, by means of energy resolution minimization. The data are then corrected for the $x$ and $y$ position dependence. Table ${ }^{9}$ ) 


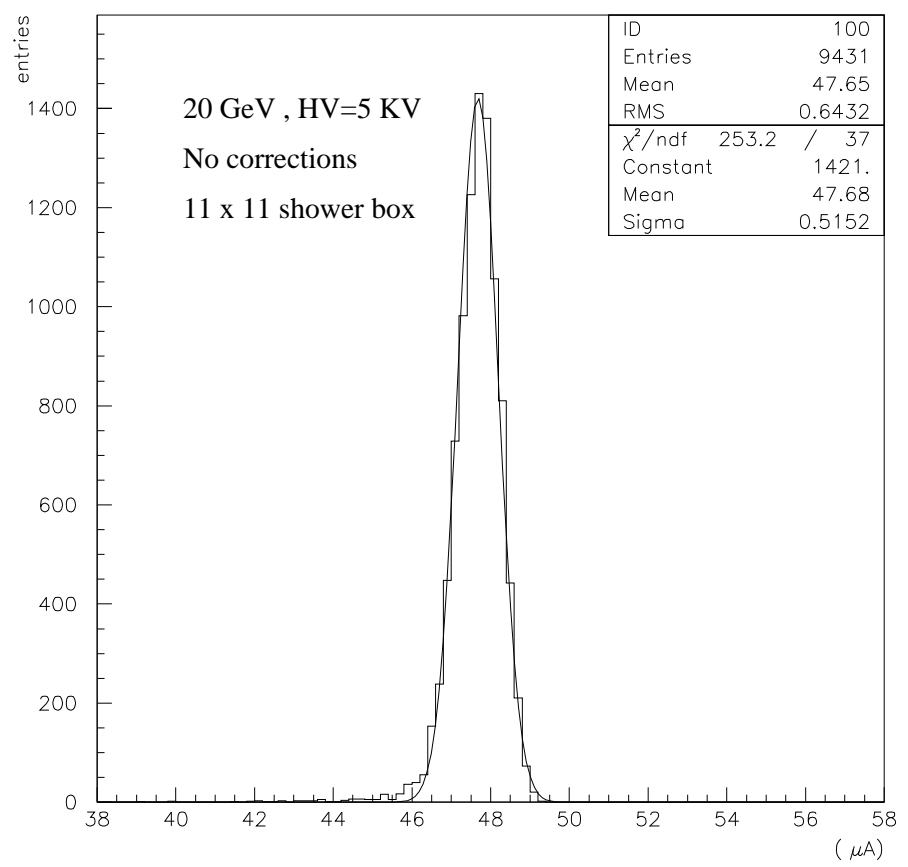

Figure 6: Pulse height spectrum for $20 \mathrm{GeV}$ electrons.

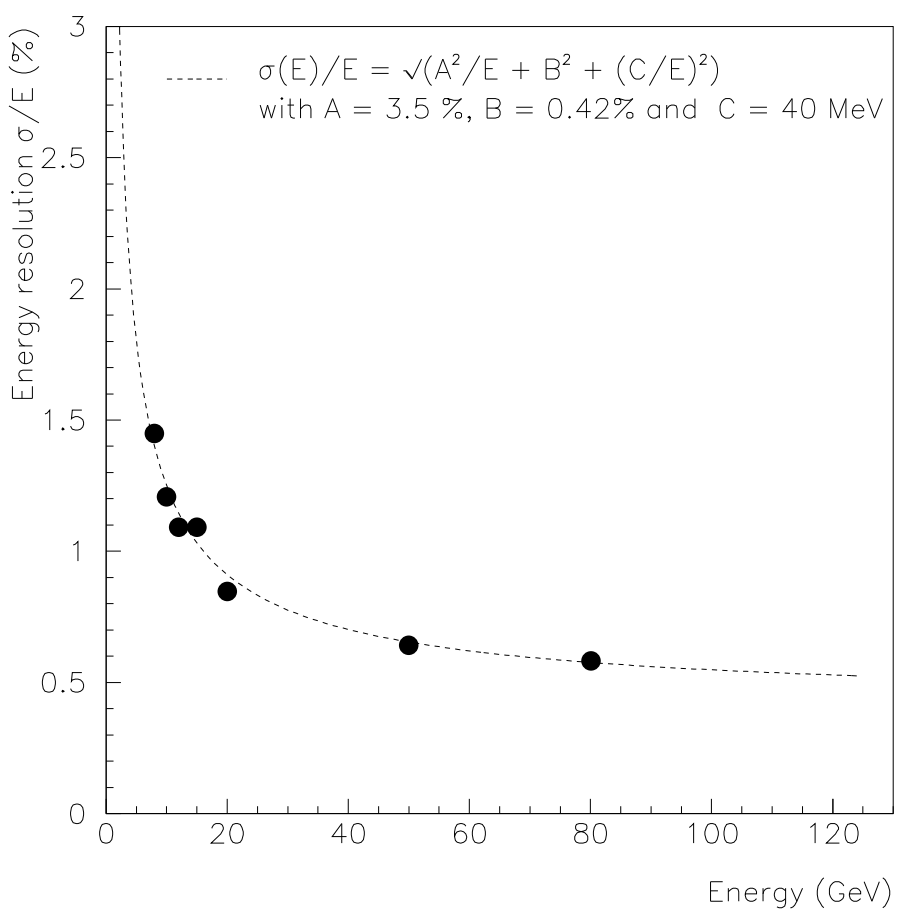

Figure 7: Energy resolution as function of the electron beam energy. 


\begin{tabular}{|c|c|c|}
\hline Energy (GeV) & Data & Monte Carlo \\
\hline & $\frac{\sigma(E)}{E}(\%)$ & $\frac{\sigma(E)}{E}(\%)$ \\
\hline 8 & $1.35 \pm 0.02$ & $1.34 \pm 0.04$ \\
10 & $1.19 \pm 0.01$ & $1.08 \pm 0.04$ \\
12 & $1.12 \pm 0.01$ & $1.01 \pm 0.04$ \\
15 & $0.97 \pm 0.01$ & $0.90 \pm 0.03$ \\
20 & $0.83 \pm 0.01$ & $0.78 \pm 0.03$ \\
35 & $0.91 \pm 0.01$ & $0.61 \pm 0.02$ \\
50 & $0.63 \pm 0.01$ & $0.52 \pm 0.02$ \\
80 & $0.57 \pm 0.01$ & $0.41 \pm 0.01$ \\
\hline
\end{tabular}

Table 1: Energy resolution for test beam and Monte Carlo data.

reports the energy resolution for Monte Carlo and test beam data. Figure 8 shows the energy resolution as a function of the beam energy. The curves are the best fits to the results. The energy resolution has been fitted, after electronics noise subtraction, by means

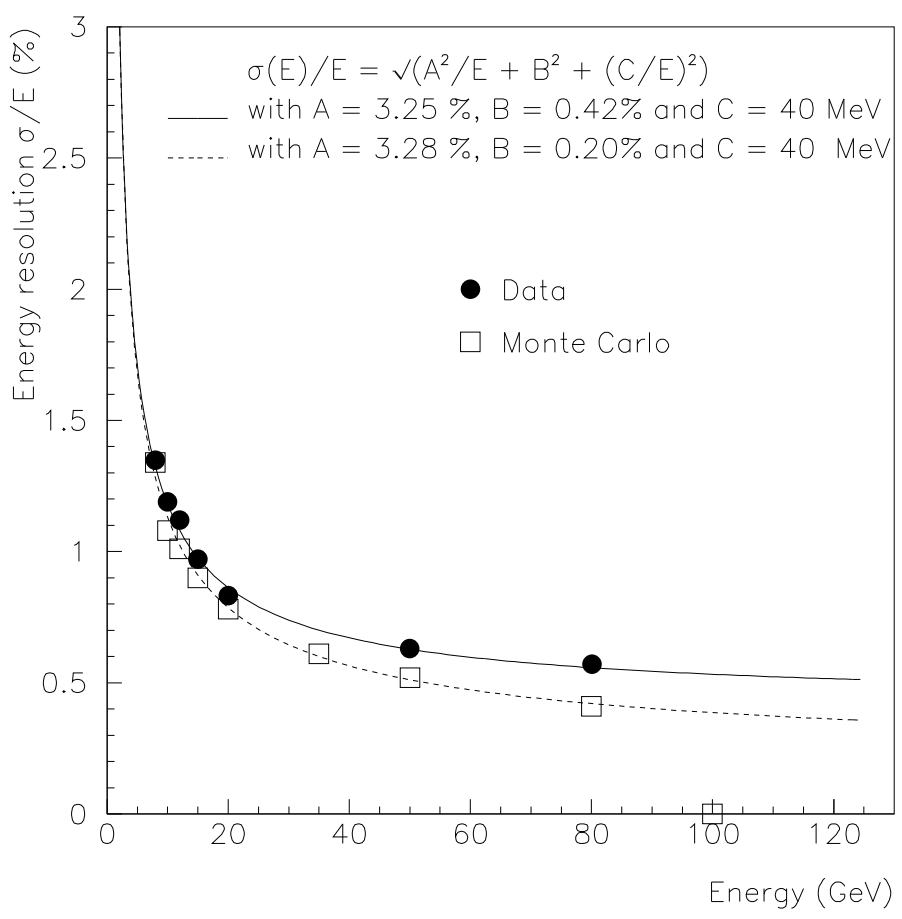

Figure 8: Comparison of Monte Carlo and beam data on energy resolution as a function of the electron energy.

of the following formula:

$$
\frac{\sigma(E)}{E}(\%)=\sqrt{\frac{A^{2}}{E}+B^{2}} .
$$

9) The $199335 \mathrm{GeV}$ result is reported for completeness, data taken in 1994 showed that the energy resolution obtained at $35 \mathrm{GeV}$ is in agreement with expectations. 
The best fit parameters are reported in table 2. There is good agreement for the energy

\begin{tabular}{|l|c|c|c|}
\hline & $\mathrm{A}$ & $\mathrm{B}$ & $\chi^{2} / n d f$ \\
\hline Data & $3.25 \pm 0.03$ & $0.42 \pm .01$ & 8.4 \\
Monte Carlo & $3.28 \pm 0.07$ & $0.20 \pm .03$ & 0.8 \\
\hline
\end{tabular}

Table 2: Best fits to the energy resolution.

dependent part of the energy resolution between Monte Carlo and test beam data, while at high energy the comparison is more difficult due to the residual constant term $(\simeq 0.4 \%)$ for the real calorimeter. A major contribution to the statistical term, A, of the energy resolution is due to the shower lateral leakage outside the shower box, accounting for about $2.5 \% / \sqrt{E}$.

\subsection{Uniformity studies}

\section{4 .1 Introduction}

Non-uniformities can in principle result from the read-out technique, from miscalibration of the electronics or from geometrical imperfections of the electrode structure. In order to study the calorimeter response uniformity on a wider scale, a larger cryostat entrance window of $20 \mathrm{~cm}$ diameter was mounted in 1993. The larger window increased the number of cells reached by the electron beam from about twelve to almost fifty. Section 5.4 .2 and 5.4.3 report the results based on the study of $\simeq 2 \times 10^{5} 50 \mathrm{GeV}$ electron showers taken with a wide beam spot. The calorimeter high voltage was set to $3 \mathrm{KV}$.

\subsubsection{Use of the test beam data to improve the intercalibration}

Within the illuminated cells, $11 \times 11$ and $7 \times 7$ shower boxes can be defined for twelve and forty-eight cells respectively. Using the calibration constants obtained by the calibration system, one obtains the energy responses for the twelve central cells with 11 $\times 11$ boxes. The energy resolution for the single cell (i.e. when only electrons with impact point contained in the cell under study are used) is good to about $0.6 \%$, but the spread of the individual twelve cell energy responses is up to $1 \%$ (R.M.S. $=0.32 \%$ ). This spread can be due to structural non-uniformities or to calibration problems. In a tower structure calorimeter, in which a substantial fraction of the shower (up to $50 \%$ ) is deposited in a single tower, the poor knowledge of the calibration constant translates directly into the cell-to-cell inter-calibration and may contribute significantly to the calorimeter constant term ${ }^{10)}$. The $7 \times 7$ box response for each of the 48 central cells is divided by the average, thus providing correction factors to the calibration constants. Such corrections are recursively applied to the calibration constants until the $7 \times 7$ cell to cell responses are equal. When the 48 correction constants are applied to an independent set of data, at the same energy, the spread of the $11 \times 11$ response for the 12 central cells is reduced to within $.2 \%$. The single cell energy resolution result does not improve. The spread of a set of correction factors is shown in figure 9. The R.M.S. is $0.64 \%$.

\subsubsection{Geometrical accuracy of the electrode structure}

The non-uniformities described in section 5.2 are common to all the cells and can be corrected for thanks to the good position resolution of the calorimeter. However inac-

10) The MC simulation has shown that a $1 \%$ error in the knowledge of the calibration constants, translates into $\mathrm{a} \simeq .4 \%$ contribution to the energy resolution constant term. 


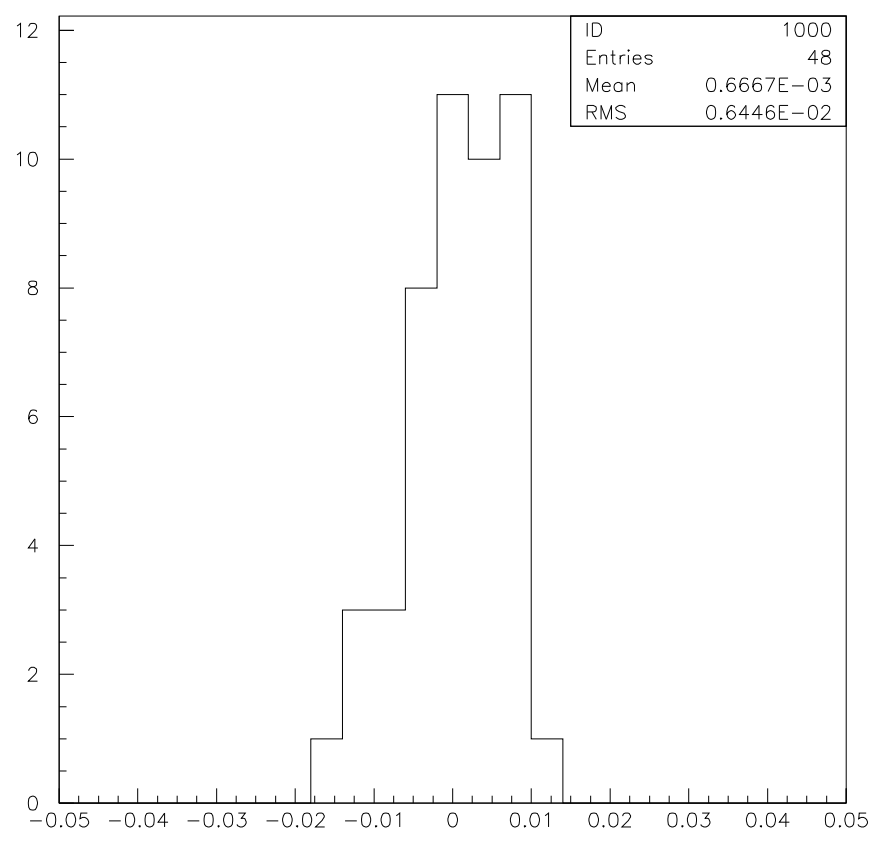

Figure 9: Spread (in \%) of the correction factors applied to the calibration constant of the 48 central cells.

curacies in the geometry (e.g. differences in the drift gap between the left and the right semi-cells) lead to non-uniformities which cannot be corrected, since they are in general different from cell to cell. As a consequence, any imperfection enters directly into the constant term of the energy resolution. To quantify such imperfections, we fit the response for each cell using the following function, where $x$ represents the position of the showering particle along the axis parallel to the drift direction as reconstructed by the calorimeter:

$$
f(x)= \begin{cases}P 1+4 P 2 \times \frac{\exp (-(x-P 3) / P 4)}{(1+\exp (-(x-P 3) / P 4))^{2}} & x \leq P 3 \\ P 5+4(P 2+P 1-P 5) \times \frac{\exp (-(x-P 3) / P 6)}{(1+\exp (-(x-P 3) / P 6))^{2}} & x>P 3\end{cases}
$$

The definition of the parameters is given in table 3 and an example of the nonuniformity is plotted in figure 10 . The difference in drift gap between two semi-cells (see section 2) is related to the value of $(\mathrm{P} 1-\mathrm{P} 5)^{11)}$. This quantity has been calculated for 35 cells illuminated by the wide-spot beam. The R.M.S. of (P1-P5) is found to be $0.26 \mu \mathrm{A}$, corresponding to $0.27 \%$ of the response. Since the simulations show that a $0.6 \%$ difference in the left/right response is explained by a displacement in the position of the collection electrode by $100 \mu \mathrm{m}$, we can estimate the magnitude of the residual imperfections:

$$
\text { R.M.S. }(\text { geometry }) \simeq \frac{0.27 \%}{0.6 \%} \times 100 \mu m=45 \mu m
$$

\footnotetext{
11) Neglecting a small contribution that can in principle be due to the miscalibration of adjacent cells.
} 


\begin{tabular}{|c|l|}
\hline Parameter & Definition \\
\hline P1 & Left response $(\mu \mathrm{A})$ \\
P2 & Depth $(\mu \mathrm{A})$ \\
P3 & Dip position $(\mathrm{cm})$ \\
P4 & Dip slope, Left $(\mathrm{cm})$ \\
P5 & Right response $(\mu \mathrm{A})$ \\
P6 & Dip slope, Right $(\mathrm{cm})$ \\
& \\
\hline
\end{tabular}

Table 3: Description of the parameters describing the response as a function of $x$.

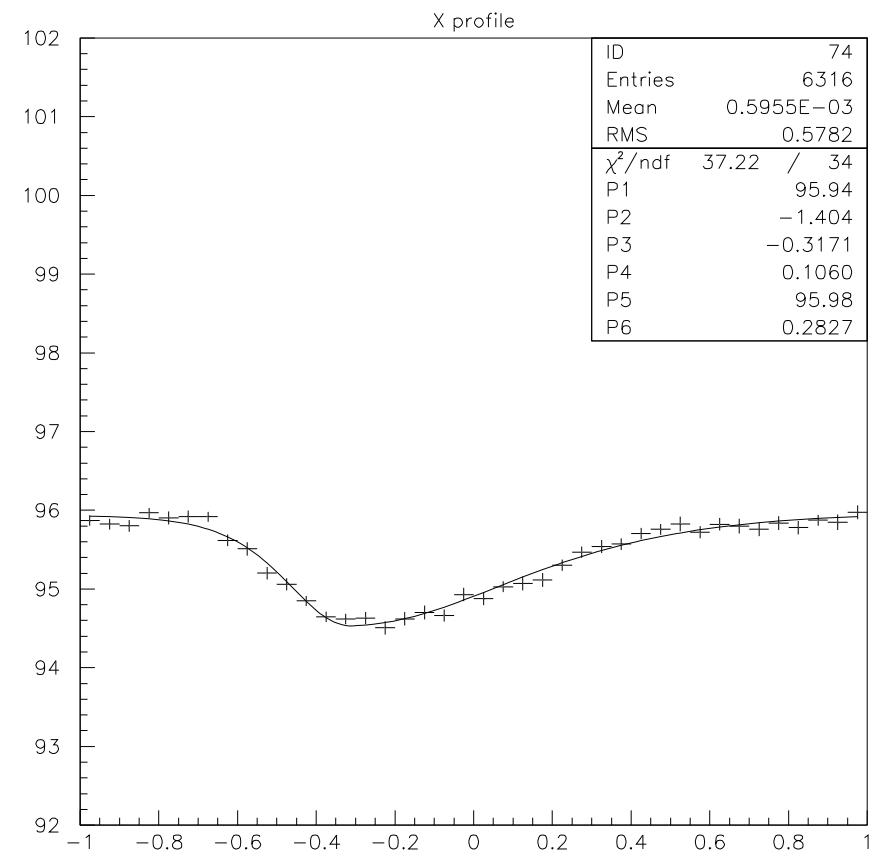

Figure 10: Description of the non-uniformity across cell 74. 


\subsection{Drift velocity}

Data collected during the test run are also used to measure the drift velocity of electrons in liquid krypton as a function of the electric field. For this purpose a delay of $5 \mu \mathrm{sec}$ was given to the flash ADC stop with respect to the trigger, and 200 samples (i.e. a time window of $5 \mu \mathrm{sec}$ ) were written on tape, rather than the 32 samples normally recorded. Figure 11 shows the typical shape of the signal for a field of $5 \mathrm{KV} / \mathrm{cm}$. The

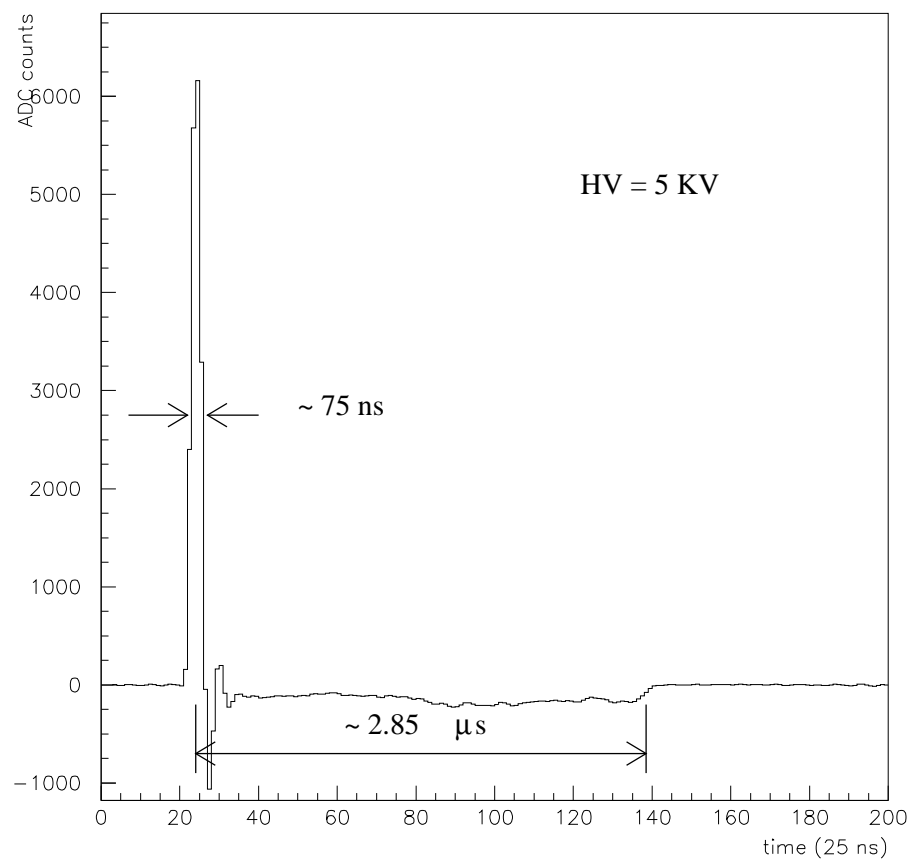

Figure 11: Typical pulse reconstructed by the prototype read out electronics.

drift time is measured as the difference between the time when the signal undershoot is reduced to half of its mean value and the time of the peak. The following cuts are applied: no saturation in both the peak and in the undershoot; the maximum must be more than 45 flash ADC counts above the pedestal in order to exclude small noisy signals; data are excluded when a non-flat undershoot is present ${ }^{12)}$. Table 4 shows the drift time and the drift velocity as a function of the high voltage at constant temperature $(\simeq 122 \mathrm{~K})$ and pressure.

In order to investigate a possible dependence of the drift velocity as a function of temperature, the liquid krypton in the calorimeter was warmed up by stopping the cooling for some time. During the warm-up, the drift velocity was measured at six different temperatures yielding a percentage variation of $-0.87 \% /(\mathrm{K})$. At a fixed temperature, no variation of the drift time as a function of the pressure is observed. The variation of current collected at the anode as a function of the temperature is explained by the drift time variation. In parallel, a more precise measurement of the temperature dependence of

12) In a few $\%$ of the events, the shower core crosses the collection electrode, yielding a concave rather than triangular current as a function of time. This creates a non-flat undershoot after the signal shaping. 


\begin{tabular}{|c|c|c|}
\hline HV $($ Volts $)$ & drift time $(\mu \mathrm{s})$ & drift velocity $(\mathrm{mm} / \mu \mathrm{s})$ \\
\hline \hline & & \\
1500 & $3.721 \pm 0.027$ & $2.687 \pm 0.020$ \\
2000 & $3.472 \pm 0.045$ & $2.880 \pm 0.037$ \\
2500 & $3.290 \pm 0.059$ & $3.040 \pm 0.054$ \\
3000 & $3.164 \pm 0.024$ & $3.160 \pm 0.023$ \\
3500 & $3.007 \pm 0.055$ & $3.326 \pm 0.061$ \\
4000 & $2.967 \pm 0.020$ & $3.370 \pm 0.022$ \\
5000 & $2.857 \pm 0.017$ & $3.500 \pm 0.021$ \\
5500 & $2.813 \pm 0.022$ & $3.555 \pm 0.028$ \\
5800 & $2.804 \pm 0.017$ & $3.566 \pm 0.022$ \\
6200 & $2.772 \pm 0.010$ & $3.608 \pm 0.013$ \\
6500 & $2.737 \pm 0.051$ & $3.654 \pm 0.068$ \\
6800 & $2.713 \pm 0.016$ & $3.686 \pm 0.022$ \\
7000 & $2.747 \pm 0.012$ & $3.640 \pm 0.016$ \\
7300 & $2.717 \pm 0.035$ & $3.681 \pm 0.048$ \\
7500 & $2.710 \pm 0.052$ & $3.690 \pm 0.071$ \\
7800 & $2.689 \pm 0.071$ & $3.719 \pm 0.097$ \\
8000 & $2.689 \pm 0.018$ & $3.719 \pm 0.025$ \\
10000 & $2.640 \pm 0.036$ & $3.788 \pm 0.053$ \\
& & \\
\hline
\end{tabular}

Table 4: Drift time and velocity as a function of high voltage.

the drift velocity was obtained using a small laser chamber[11]. Figure 12 shows the drift velocity temperature dependence for three electrical fields of interest.

\subsection{Time Resolution}

\subsubsection{Prototype electronics}

In 1994, part of the data-taking period was dedicated to test a prototype version of the final read-out electronics. During this time, eight central cells of the calorimeter were equipped with the prototype electronics. The cold preamplifiers inside the cryostat were modified to obtain a time constant of $500 \mathrm{~ns}$, shorter than the drift time of the electrons in the gap of $\simeq 3 \mu \mathrm{s}$. The preamplifier signals were connected via coaxial cables ( $2 \mathrm{~m}$ long, $50 \Omega$ impedance) to intermediate amplifiers (transceivers) mounted directly on the signal feedthroughs outside the cryostat. The transceivers had a voltage gain of about 3 with a pole zero compensation and an additional 20 ns differentiation in order to restore the triangular shape and fast rise-time of the original current signal (at the input of the preamplifier). The differential transceiver output signals were sent, via $15 \mathrm{~m}$ long shielded twisted pair cables (100 $\Omega$ impedance), to the calorimeter pipeline digitizer (CPD) modules installed in the electronics barrack.

The eight channel CPD prototype consists of a motherboard equipped with eight daughter cards. Each CPD daughter card contains a $20 \mathrm{~ns}$ differential receiver, a $4^{\text {th }}$ order $10 \mathrm{~ns}$ Bessel filter, a gain switching amplifier, a 10 bit FADC and a $200 \mu$ s pipeline storage. In order to increase the dynamic range of the system by a factor of sixteen, the gain of the switching amplifier is chosen according to the actual pulse height of the incoming pulse. The selection of the proper gain range, among the four available, is done in parallel to the 


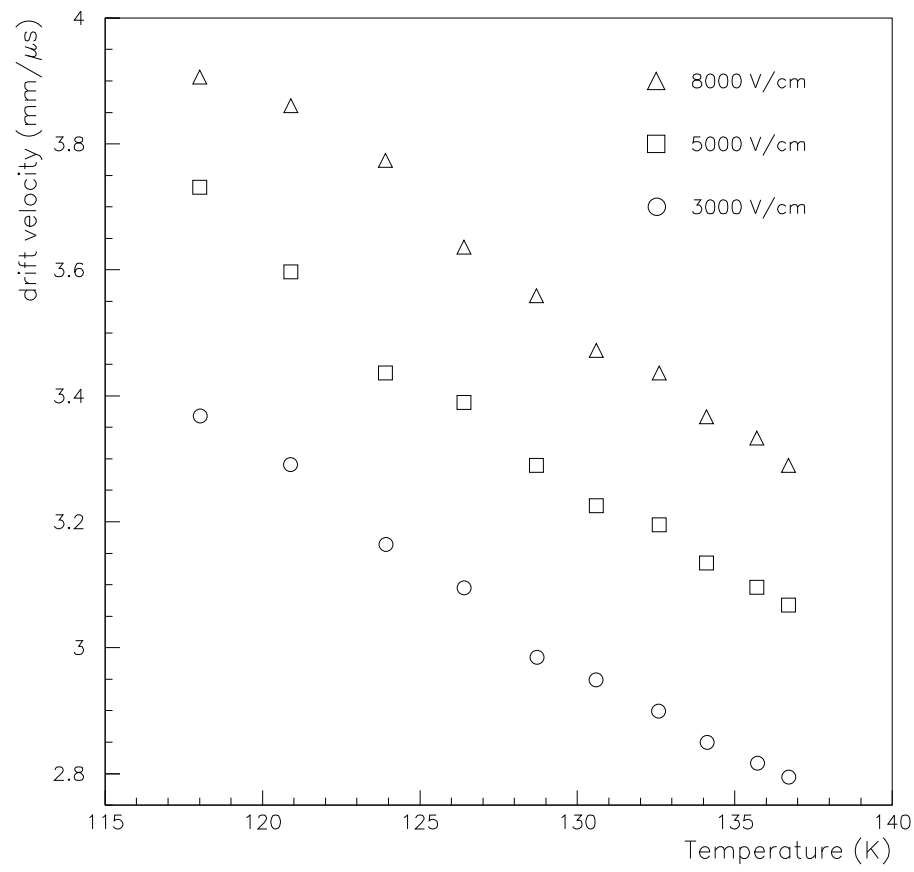

Figure 12: Drift time temperature dependence measured using the laser chamber.

pulse processing in the Bessel filter. In the final version the Bessel filter, the comparator stage and the gain-switching amplifier will be contained in an ASIC. The shaped signal is bipolar with a peaking time of $50 \mathrm{~ns}$ and has a flat undershoot. The undershoot is as long as the electron drift time and has an amplitude equal to about $3 \%$ of the maximum pulse height.

The data, digitized by a $40 \mathrm{MHz} \mathrm{FADC}^{13}$, are read from the pipeline memory by a RISC processor ${ }^{14)}$ with a speed of $10 \mathrm{MHz}$ per sample. Another RISC processor of the same type is used to control the read-out. This trigger interface and control (TIC) module sends the trigger and controls the memories of the CPD. The TIC is also used to download the thresholds for the gain switching circuit and the number of time samples to be transmitted.

Offline, the signal is reconstructed from the sampled data. Both the time and the amplitude of the signal are determined. It is of extreme importance for the experiment to have a precise time information, since this is used to verify that the signal is produced by an event with a valid trigger and to distinguish between $K_{L}$ and $K_{S}$ events. The time resolution has to be very good in order to allow a narrow coincidence with the signal from the tagging counter [3], placed on the proton beam originating the $K_{S}$ particles.

\subsubsection{Results}

To determine the time and amplitude of the calorimeter pulses, a digital filter[12] is used offline. The method has also been used successfully by other groups[13]. The filter assigns a set of weights for each data sample, derived from the pulse itself. Separated sets

13) SPT7824, by SPT, Colorado Springs, Colorado 809907, US.

14) RIO 8260, by CES, CH1213 Petit-Lancy, Switzerland. 
of weights are used for the time and for the amplitude measurements. Each data sample is multiplied by the corresponding weight and the sum of the multiplications gives the final value:

$$
\begin{aligned}
& A=\Sigma w_{A_{i}} \times S_{i} \\
& \tau=\frac{1}{A} \Sigma w_{\tau_{i}} \times S_{i}
\end{aligned}
$$

The weights $w_{A_{i}}$ and $w_{\tau_{i}}$ are calculated using a program [14] developed for applications in the framework of the large hadron collider (LHC). For the determination of the time resolution described here, only the time of the pulse was determined by the digital filter method. The weights are determined from a subsample of the data, using the time measurement provided by a scintillation counter. In order to normalize the pulses to the same height, a parabola fit was used to measure the amplitudes. A 50 ps resolution TDC ${ }^{15)}$ was used to measure the phase between the trigger, and hence the pulse, and the $40 \mathrm{MHz}$ FADC clock. The final time resolution is then determined applying the resulting weights to an independent data sample not used in the training procedure.

The time distribution after the filter application is shown in figure 13 for $50 \mathrm{GeV}$ data. Seven samples are used encompassing the full pulse shape, and no additional corrections

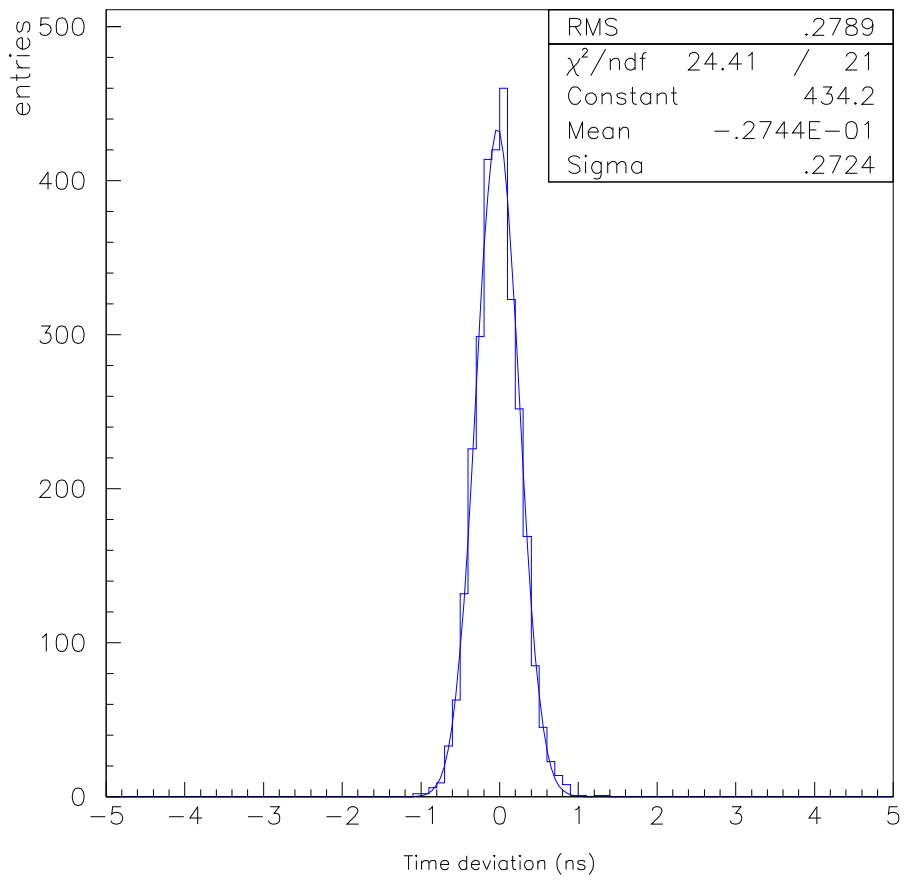

Figure 13: Time distribution at $50 \mathrm{GeV}$.

or cuts are applied. The width at half maximum of the distribution is 272 psec. Figure 14 shows the energy dependence of the time resolution where the contribution of the scintillation counter (measured to be about 180 psec) is quadratically unfolded.

15) $2228 \mathrm{~A}$, LeCroy Corporation. 


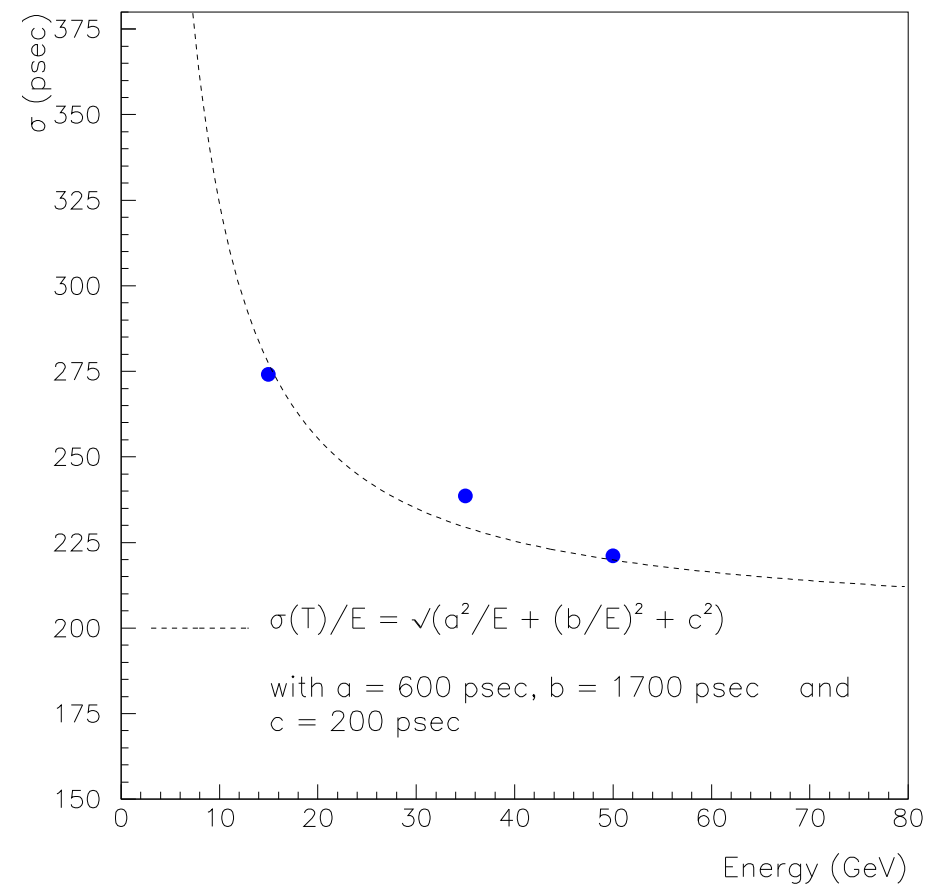

Figure 14: Energy dependence of the time resolution ( a trigger resolution of 180 ps has been subtracted in quadrature).

The time resolution is also determined from the rising edge of the pulse using a parabola fit to determine the maximum amplitude. Using this method, several corrections, all predicted by the Monte Carlo simulation, are needed. These corrections include the impact point dependence of the shower within the drift gap, the correlation of the phase with the sampling clock and the time slewing effect. The two analyses yield similar results.

\section{$6 \quad$ Response at high intensity}

Most of the data were collected with beam conditions corresponding to an energyflow into the front face of the calorimeter in the range of 150 to $1500 \mathrm{GeV} \mathrm{cm}^{-2} \mathrm{~s}^{-1}$. These values are well representative of the running conditions planned for the NA48 experiment. In this range of intensity, no significant variations of response of the detector are observed.

In a previous prototype test [4] it was observed that the detector response deteriorated at higher intensities. This was found compatible with expectations based on a model which considered the distortion of the electric field due to the space-charge of positive ions. To confirm this effect, new data at high intensity and at two values of the calorimeter high voltage have been taken.

Using a wide-spot beam which covered about 30 cells of our detector, we took data with intensities as high as $6500 \mathrm{GeV} \mathrm{cm}^{-2} \mathrm{~s}^{-1}$. For small beam-spot runs, when the beam impinges on a single cell, the effective intensity, taking into account the transverse spread of the energy deposition in a shower, reached $\simeq 30000 \mathrm{GeV} \mathrm{cm}^{-2} \mathrm{~s}^{-1}$.

The most relevant feature of the data collected at high intensity is a drop of the response for showers with maximum energy deposition in the proximity of an anode. We observe an effect as large as $6 \%$ with the high voltage set at $3 \mathrm{kV}$. This drop is reduced by 
a factor of about 2.5 when the high voltage is set to $5 \mathrm{kV}$. This reduction is in agreement with the space-charge model, which predicts that it should be about $(5 / 3)^{2}=2.8$. This prediction is based on two facts. Firstly, the space-charge density is inversely proportional to the drift velocity of the ions, and therefore inversely proportional to the voltage. Secondly, the distortion of the electric field is proportional to the ratio of the space charge density to the electrode surface charge density at low intensity, the latter being proportional to the high voltage. Deviations from this simple prediction are due to saturation in the dependence of the drift speed on the electric field. Further evidence in favor of the space-charge model comes from the observation that the response drop grows in the first $0.5 \mathrm{~s}(0.75 \mathrm{~s})$ of each SPS burst, at $5 \mathrm{kV}(3 \mathrm{kV})$. This is the time necessary to approach the equilibrium distribution of space-charge, which is then maintained during the rest of the burst, and disposed during the interval between bursts. The corresponding mobility of the ions is $\simeq 0.5 \mathrm{~cm}^{2} \mathrm{~s}^{-1} \mathrm{kV}^{-1}$. The value of the space-charge density at equilibrium is in agreement with the magnitude of the response drop for shower axes near the anode.

\section{Acknowledgements}

We gratefully acknowledge the effort of J. Giacometti and J. Vergnaud for the drawing of the calorimeter, M. Arnaud, J. Armand, D. Dattola, G. Decock, T. DeOliveira, B. Duboue, G. Dughera, G. Fersurella, G. Juban, J. Habault, P. LeCossec, M. Melchiorri, E. Pasquetto, R. Ruberti, D. Therville, R. Truhan, and W. Wilkens for the construction and installation of the prototype calorimeter, F. Bal, G. Pagani, A. Papi, J.C. Tarle, L.Zaccarelli for the preparation of the front-end and read-out electronics and the contribution of F. Louis, for setting up the chambers. We thank all our NA48 colleagues for their help during setup and data taking. We thank the technical support groups in AT, ECP, PPE and SL divisions at CERN for their help.

\section{References}

[1] G.D. Barr et al., CERN/SPSC/90-22/P253

[2] H.Burkhardt et al., NIM A 268 (1988) 116;

H.Burkhardt et al., PL B206 (1988) 169;

G.D.Barr et al., PL B317 (1993) 233.

[3] P. Grafström et al.,NIM A 344 (1994) 487.

[4] V. Fanti et al., NIM A 344 (1994) 507.

[5] C.Cerri et al., NIM 227 (1984) 227.

[6] B.Aubert et al., CERN/DRDC/90-31 DRDC/P5.

[7] B.Hallgren, G.Laverrière, CERN/ECP 91-33.

[8] B.Gorini, diploma thesis, Pisa, 1993.

[9] C. Cerri et al., in preparation.

[10] P.Debu and E. Mazzucato NA48 Note 93/18.

[11] A. Kalinin et al., to be published.

[12] W.E.Cleland and E.G.Stern, NIM A 338 (1994) 467.

[13] O.Benary et al., NIM A 349 (1994) 367.

[14] S.J.Inkinen, FIROSMIN 3.2, CERN-ECP-RD12 94. 